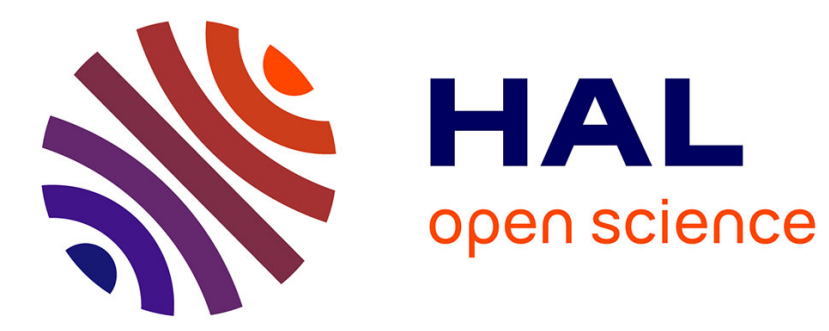

\title{
Dependence of time reversal of acoustic waves in plates on mean frequency and plate's characteristics
}

\author{
H. Zophoniasson, C. Hudin, C. Bolzmacher, M. Hafez
}

\section{To cite this version:}

H. Zophoniasson, C. Hudin, C. Bolzmacher, M. Hafez. Dependence of time reversal of acoustic waves in plates on mean frequency and plate's characteristics. Microsystem Technologies, 2016, 22 (7), pp.1791-1798. 10.1007/s00542-016-2834-2 . hal-01845530

\author{
HAL Id: hal-01845530 \\ https://hal.science/hal-01845530
}

Submitted on 8 Jan 2019

HAL is a multi-disciplinary open access archive for the deposit and dissemination of scientific research documents, whether they are published or not. The documents may come from teaching and research institutions in France or abroad, or from public or private research centers.
L'archive ouverte pluridisciplinaire HAL, est destinée au dépôt et à la diffusion de documents scientifiques de niveau recherche, publiés ou non, émanant des établissements d'enseignement et de recherche français ou étrangers, des laboratoires publics ou privés. 


\title{
Dependence of time reversal of acoustic waves in plates on mean frequency and plate's characteristics
}

\author{
Harald Zophoniasson $^{1} \cdot$ Charles Hudin $^{1} \cdot$ Christian Bolzmacher $^{1} \cdot$ Moustapha Hafez $^{1}$
}

\begin{abstract}
Time reversal is an approach that can be used to focus acoustic waves in a particular location on a surface, allowing a multitouch tactile feedback interaction. The spatial resolution in this case depends on several parameters, such as geometrical parameters, injected frequency and material properties. This can be efficiently described with the Kirchhoff model of wave propagation. This paper highlights the impact of frequency, in particular the mean frequency of the injected signal band, geometrical parameters such as plate thickness and transducer's surface on the focused spot dimension and displacement amplitude. Results show that the dimension of piezoelectric transducers has little influence on the spatial resolution. The resonant behavior of the plate and its implication on focus point dimension, in particular its shape and amplitude are investigated. On reduced bandwidths the predominant effect of the resonant frequencies on the amplitude and shape of the focus spot is shown.
\end{abstract}

\section{Introduction}

Touch interaction has become the main interaction modality with most mobile devices as it allows the user to interact naturally with simple and intuitive gestures. Touchscreens however lack realism and efficiency compared to physical keyboards or buttons (Hoggan et al. 2008). Adding haptic feedback addresses this problem. Simple solutions relying on small motors with a rotating eccentric mass provide

Harald Zophoniasson

harald.zophoniasson@cea.fr

1 CEA, LIST, Sensorial and Ambient Interfaces Laboratory, 91191 Gif-sur-Yvette Cedex, France poor tactile feedback (Mortimer et al. 2007). Linear electromagnetic actuators (Kildal 2012) and other technologies such as piezoelectric ceramics (Chen et al. 2011; Poupyrev et al. 2002) are able to provide richer interactions due to their larger frequency range. However, as vibrations are conveyed to the whole surface (not localized), multi feedback (i.e. presenting different haptic feedback to different touch locations simultaneously) is not possible with such approaches.

Time reversal of flexural waves has been previously implemented on thin plates in (Hudin et al. 2013), to address the multi-feedback challenge. The process relies on the focusing of flexural waves in a solid material. It generates a constructive interference of flexural waves produced by actuators located on the perimeter of the surface. This allows the production of multiple localized vibrations simultaneously. The choice of a transparent material makes time reversal compatible with any kind of visual display.

The spatial resolution of such a system is a crucial parameter in the design of a tactile feedback interface. According to (Hudin et al. 2012) the spatial resolution is bounded by the smallest wavelength, $\lambda-=\mathrm{c} / \mathrm{f}+$, where $\mathrm{c}$ is the medium's wave propagation celerity, and $\mathrm{f}+$ the highest frequency used in the re-focusing process. Time reversal has already been implemented on several materials, such as BoPET (Biaxially-oriented polyethylene terephthalate) (Hudin et al. 2012) and glass (Hudin et al. 2013), which were in fact very thin membranes. However in order to implement it on touchscreens for tablet and even larger interactive screens, time reversal approach needs to be validated on thicker and larger surfaces. This paper addresses this issue.

After a brief explanation of the time reversal principles, we present an analytical model based on Kirchhoff's equation for wave propagation to compute the spatial resolution 
of time reversal of flexural waves applied to plates. Measurements on a physical system are then confronted to the model prediction. A method to evaluate the material characteristics of the plate is introduced to adjust the model to the physical system. The system's resonant behavior is finally analyzed to propose an explanation for shape and height variations of the focalization point.

\section{Principles of operation}

An impact at a specific location on a plate generates a wave field in the plate. The Fresnel-Kirchhoff theorem states that this wave field can be entirely retrieved from the value of the solution and from that of its first derivative on a surface enclosing it. To simplify, an approximation of this wave field can be recorded at a finite set of locations on the boundary of the plate (Fink 1992; Wu et al. 1992). Time reversal consists in recording the wave propagation speed after an impact at one or more locations on the perimeter of the plate. Playing the time reversed signal at the recording locations, i.e. generating the displacements will result in the back propagation of the waves until the original point of impact, recreating an approximation of the displacement at the impact's point. For in-depth description of the phenomenon see Fink and Prada 2001.

The generation of the driving signals of the actuators follows the method presented in (Hudin et al. 2013), to avoid the complex tasks of creating calibrated and repeatable impacts at specific locations on a plate. Based on the reciprocity principle and the fact that the wave propagation equation is linear, one can generate impulses at the transducer locations and record the vibration at the focalization point. Instead of a mechanical impulse, a voltage impulse on the transducer is theoretically sufficient. A voltage impulse is however brief and injects too little energy in the plate which leads to noisy measurements and can be replaced by white noise as described in Eq. (1), with $h_{i, j}(t)$, the impulse response at the focus point $j$ due to the impulse on actuator $i, \mathrm{FT}^{-1}$ the inverse Fourier transform, $\hat{\mathrm{U}}_{\mathrm{j}(\omega)}$ the recorded displacement at point $\mathrm{j}$ and $\mathrm{V}_{\mathrm{i}}$ the white noise signal driving actuator $i$.

$h_{i, j}(t)=F T^{-1}\left[\frac{\hat{U}_{j(\omega)}}{\hat{V}_{i(\omega)}}\right]$

\section{Analytical model}

The displacement around the point of focalization $u$, located in $r=0$, is described as superposition of plane waves (modeled by a Bessel function $J_{0}$ ) of amplitude $A$, angular velocity $\omega$ and wavenumber $k$ coming from every space direction and interfering additively in $O$ (Cassereau and Fink 1992; Hudin 2014). $T$ is the time of the focalization.

$u(r, t)=\int_{\omega} A(\omega) J_{0}(k r) e^{j \omega(t-T)} d \omega$

Spatial resolution $R_{S}$ is given by the width at halfheight of the focus point at the time of focalization, $u\left(\frac{R_{s}}{2}, t=T\right)=\frac{1}{2} u(0, t=T)$. For a constant bandwidth between pulsation $\omega_{1}$ and $\omega_{2}, A(\omega)=1, \forall \omega \in\left[\omega_{1}, \omega_{2}\right]$, so:

$$
\int_{\omega=\omega_{1}}^{\omega_{2}} J_{0}\left(k R_{s} / 2\right) d \omega=\frac{1}{2} \int_{\omega=\omega_{1}}^{\omega_{2}} J_{0}(0) d \omega=\frac{1}{2}\left(\omega_{2}-\omega_{1}\right)
$$

Taking the limited development of the second order Bessel function around $O, J_{0}(x)=1-\left(\frac{x}{2}\right)^{2}$ and the dispersion relation in a thin plate $\omega=\sqrt{\frac{D}{\rho_{s}}} k^{2}$ from the propagation model of waves in plates by Kirchhoff, gives:

$\omega_{2}-\omega_{1}-\frac{1}{16} \sqrt{\frac{\rho_{s}}{D}} R_{s}^{2}\left(\frac{\omega_{2}^{2}}{2}-\frac{\omega_{1}^{2}}{2}\right)=\frac{1}{2}\left(\omega_{2}-\omega_{1}\right)$

which is,

$R_{s}^{2}=16 \sqrt{\frac{D}{\rho_{s}}} \frac{\omega_{2}-\omega_{1}}{\omega_{2}^{2}-\omega_{1}^{2}}=8 \sqrt{\frac{D}{\rho_{s}}} \frac{2}{\omega_{2}+\omega_{1}}$

$R_{s}=\sqrt[4]{\frac{D}{\rho_{s}}} \sqrt{\frac{8}{\omega_{m}}}=\sqrt[4]{\frac{D}{\rho_{s}}} \sqrt{\frac{8}{2 \pi f_{m}}}$

with $\omega_{m}=\frac{\omega_{2}+\omega_{1}}{2}$ the mean angular velocity, $D=\frac{E(2 h)^{2}}{12\left(1-\nu^{2}\right)}$ the bending rigidity and $\rho_{s}=\rho \times 2 h$ the density per square meter. By injecting $\omega_{m}=\sqrt{\frac{D}{\rho_{s}}} k^{2}, k=\frac{2 \pi}{\lambda_{m}}$ into Eq. (6), the spatial resolution can be expressed as a function of the mean wavelength $\lambda_{m}$ :

$R_{s}=\frac{\lambda_{m} \sqrt{8}}{2 \pi} \approx \frac{\lambda_{m}}{2.2}$

With Eq. (6) the model links the spatial resolution to material properties contained in the terms $D$ and $\rho_{s}$ and to the mean wavelength of the actuation bandwidth used. The mechanical properties for standard glass are: $E=69 \mathrm{GPa}$, $v=0.25$ and $\rho=2500$. Equation (7) explicitly points out the relation between the spatial resolution and the mean wavelength induced by the excitation bandwidth.

Without the knowledge of the precise material properties a well-known method was used to estimate them (Aki 1957; Boschi et al. 2013). It relies on the measure of surface wave velocity through the correlation of the recording in different location of the plate of the same signal. The 
Table 1 Computed value for $\alpha$ for each plate thickness

\begin{tabular}{lll}
\hline Plate thickness & $2 \mathrm{~mm}$ & $3 \mathrm{~mm}$ \\
\hline$\alpha$ & 0.5434 & 0.4606 \\
\hline
\end{tabular}

correlation $C_{p q}$, in the spectral domain between two signals recorded in $p$ and $q$ is given by:

$C_{p q}(\omega)=\frac{S_{p}(\omega) \cdot S_{q}^{*}(\omega)}{\left|S_{p}(\omega)\right|\left|S_{q}(\omega)\right|}$

where $S_{q}^{*}(\omega)$ is the complex conjugate of $S_{q}(\omega)$. From Aki (1957) and Boschi et al. (2013), we have:

$\mathbb{E}\left[\Re\left(C_{p q}(\omega)\right)\right]=J_{0}\left(\frac{\omega r_{p q}}{V_{\varphi}(\omega)}\right)=J_{0}\left(\alpha \sqrt{\omega} r_{p q}\right)$

With $\mathbb{E}$ the expected value, $\Re$ the real part and $V_{\varphi}(\omega)$ the phase speed of the waves at angular velocity $\omega$. This phase speed in thin plates is given by:

$V_{\varphi}(\omega)=\sqrt[4]{\frac{D}{\rho_{s}}} \sqrt{\omega}=\frac{1}{\alpha} \sqrt{\omega}$

For a group of angular velocity $\omega$ and pairs $\{p, q\}$, the coefficient $\alpha$ is the one which minimizes the quantity $\left(\Re\left(C_{p q}(\omega)\right)-J_{0}\left(\alpha \sqrt{\omega} r_{p q}\right)\right)^{2}$,

$\alpha=\arg _{\xi} \min \left[\sum_{\omega} \sum_{p} \sum_{q \neq q}\left(\Re\left(C_{p q}(\omega)\right)-J_{0}\left(\alpha \sqrt{\omega} r_{p q}\right)\right)^{2}\right]$

To estimate the material properties, a signal was emitted and recorded at different locations. Those recordings were used to compute the correlation term. Inserted into Eq. (11), an estimate of $\alpha$ can be found, which is, according to Eq. (10) an estimate of $\sqrt[4]{\frac{\rho_{s}}{D}}$. This value represents an aggregate of the material properties for each plate thickness shown in Table 1. Injected into Eq. (6) an adjustment to the proposed model was computed.

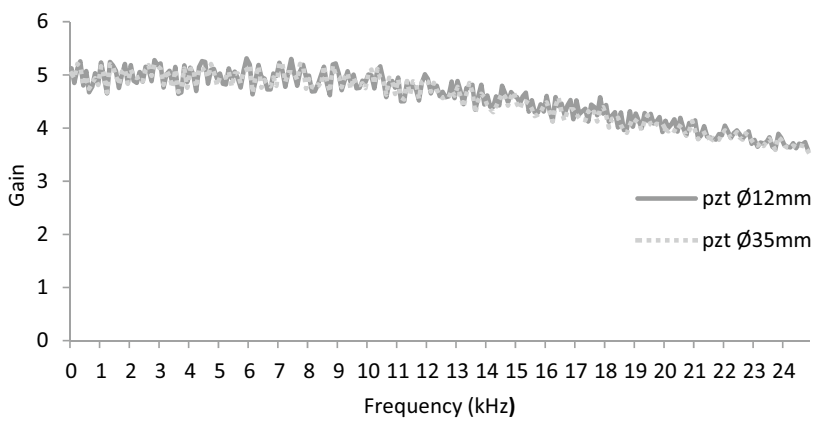

Fig. 2 Gain of the amplification stage for the $0-25 \mathrm{kHz}$ bandwidth

\section{Device setup}

Rectangular glass plates of dimensions $330 \times 254 \mathrm{~mm}^{2}$ with thicknesses of $2 \mathrm{~mm}$ and $3 \mathrm{~mm}$ are used in the set-up. The plates are laid on 4 simple supports and free on each border. The first resonant mode was measured at 41.5 and $54 \mathrm{~Hz}$ for the 2 and $3 \mathrm{~mm}$ plates, respectively. The transducers used are piezoelectric diaphragms, (Murata 7BB12-9 and 7BB-35-3), of diameter 12 and $35 \mathrm{~mm}$ and with thickness of 0.12 and $0.23 \mathrm{~mm}$ respectively. 16 transducers (8 on each side, in pairs facing each other) are glued with epoxy resin on the glass plates at a distance of $10 \mathrm{~mm}$ from the edges. An uneven distribution as shown in Fig. 1 aims at avoiding any symmetrical arrangements that would give correlated waveforms. The 16 transducers are driven in pairs in order to maximize the plate's deformation.

A total of four plates are prepared, two $2 \mathrm{~mm}$ thick and two $3 \mathrm{~mm}$ thick plates. One of the $2 \mathrm{~mm}$ thick plates is equipped with $12 \mathrm{~mm}$ transducers, the other one, with $35 \mathrm{~mm}$ transducers. The same is done for the $3 \mathrm{~mm}$ thick plates.

The acquisition and control software is developed in LabVIEW. Command signals (sample frequency $50 \mathrm{kHz}$, giving a bandwidth of $25 \mathrm{kHz}$ ) are amplified to $30 \mathrm{Vcc}$ through a custom board employing OPA552 (gain set to 5) operational amplifier chips. The plate's displacements are measured with a laser vibrometer (Polytec OFV 534). The
Fig. 1 Position of the piezoelectric transducers on the glass plate
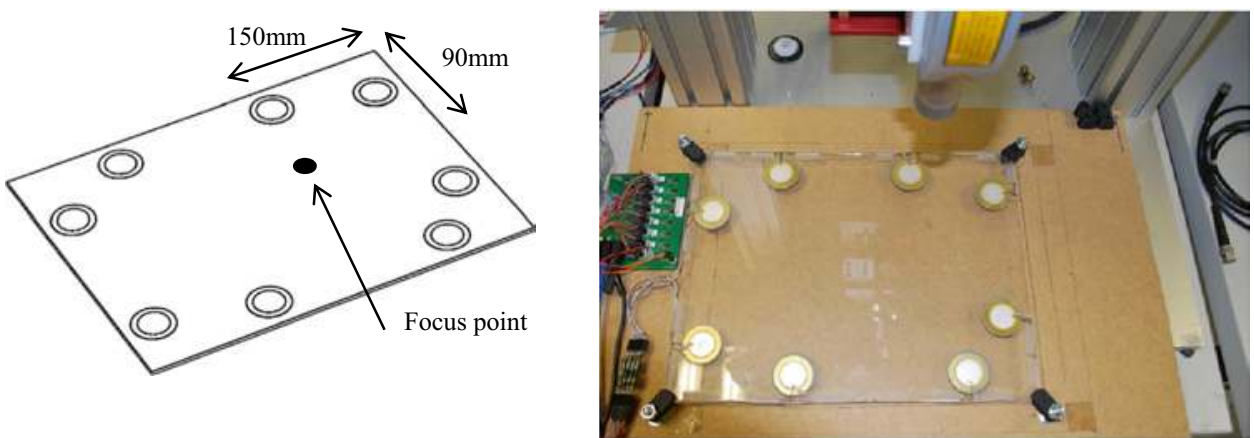
Fig. 3 Displacement magnitude $(\mu \mathrm{m})$ at the focus point for a $25 \mathrm{kHz}$ wide bandwidth and A-B cross section detail
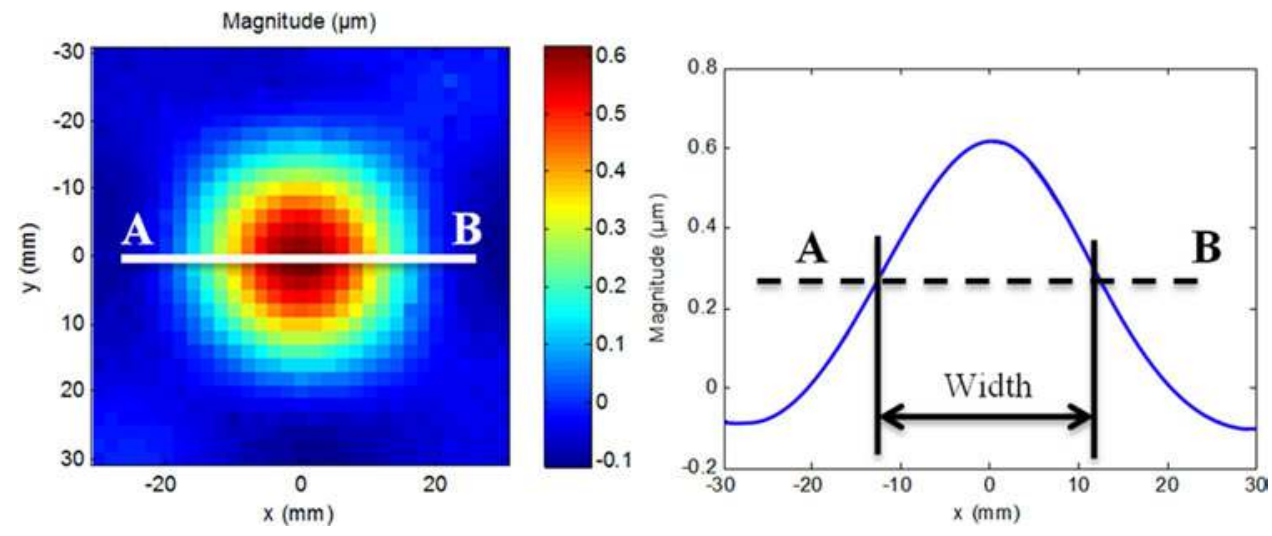

Table 2 Width (mm) and height $(\mu \mathrm{m})$ of focus point

\begin{tabular}{llllll}
\hline Width $(\mathrm{mm})$ & PZT Ø35 mm & PZT Ø12 mm & Height $(\mu \mathrm{m})$ & PZT Ø35 mm & PZT Ø12 mm \\
\hline Glass $2 \mathrm{~mm}$ & 23.7 & 22.2 & Glass $2 \mathrm{~mm}$ & 3.59 & 0.61 \\
Glass $3 \mathrm{~mm}$ & 23.1 & 25.7 & Glass $3 \mathrm{~mm}$ & 2.78 & 0.51 \\
\hline
\end{tabular}

performance of the specifically built amplification stage is tested. Due to stability problems when driving the highly capacitive charge of PZT (lead zirconate titanate ceramic material) transducers $(12 \mathrm{~mm}: 8 \mathrm{nF}, 35 \mathrm{~mm}: 30 \mathrm{nF}$ at $1 \mathrm{kHz}$ ), a hardware low pass filter was added on the amplification stage. Gain and phase were measured across the entire bandwidth $(0-25 \mathrm{kHz})$. Figure 2 shows the transfer function with an almost constant gain of 5 between 0 and $8 \mathrm{kHz}$. At about $8-9 \mathrm{kHz}$ the gain slowly decreases to reach 3.6 at $25 \mathrm{kHz}$, a decrease of $28 \%$.

\section{Experiments}

A first study aims at determining the effect of plate's thickness and PZT transducers surface on the dimension of the focus point while using the whole bandwidth $(0-25 \mathrm{kHz})$ of the system. For each one of the four plates, the drive signals are computed for the coordinates $(150 \mathrm{~mm} ; 90 \mathrm{~mm})$ on the plate as shown in Fig. 1. To obtain a complete recording of the wave's propagation around the focus point in time, measurements of the vibrations are taken along a grid of $60 \times 60 \mathrm{~mm}$ with a $2 \mathrm{~mm}$ step size, centered on the focus point as shown in Fig. 3. This allows to reconstruct the propagation of the wave in the glass plate. The duration of focalization is set to $2 \mathrm{~ms}$. The corresponding timeframe is used to measure the width of the focus point. The width of the focus point is defined as its width at half-height as shown in Fig. 3. The measurement is done along the cross section $A B$.

Across the four experiments the width of the focus point is relatively constant, at about $23 \mathrm{~mm}$, as shown in Table 2 . The measurements for the $35 \mathrm{~mm}$ PZT transducer exhibit a really stable width, whilst those for the $12 \mathrm{~mm}$ PZT transducer show a slight variation. The height of the focus point decreases with the increase of the plate's thickness from 2 to $3 \mathrm{~mm}$ ( $-26 \%$ for the $35 \mathrm{~mm}$ PZT and $-16 \%$ for the $12 \mathrm{~mm}$ PZT), as shown in Table 2 . This is easily explained by the fact that energy input remains the same while the stiffness of the plate increases with its thickness.

To better understand the relation of the focus point's width with the other parameters, we measured it for different frequency bands, plate thicknesses and actuator size. For plate thicknesses of 2 and $3 \mathrm{~mm}$ and for 12 and $35 \mathrm{~mm}$ wide piezoelectric diaphragms, the time reversal process on $1 \mathrm{kHz}$ signal bands beginning from $0-1 \mathrm{kHz}$ up to $24-25 \mathrm{kHz}$ is used.

\subsection{Focus point's width}

Figure 4 present the results for each frequency band compared to the spatial resolution computed with the analytical

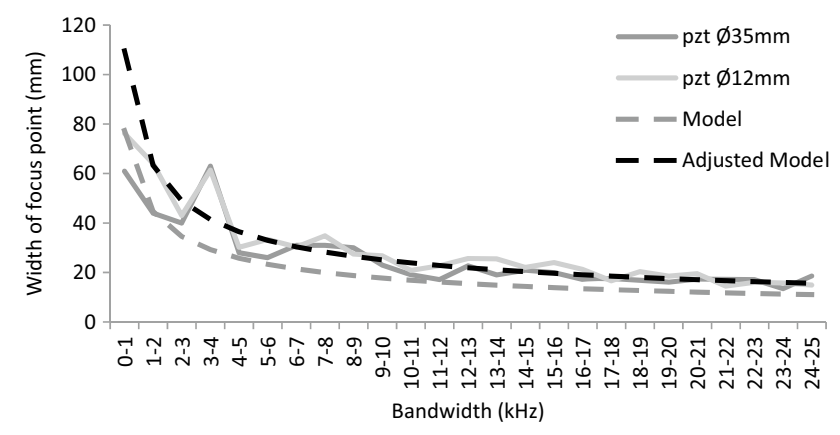

Fig. 4 Model and measurements of focus point's width on a $3 \mathrm{~mm}$ glass plate 


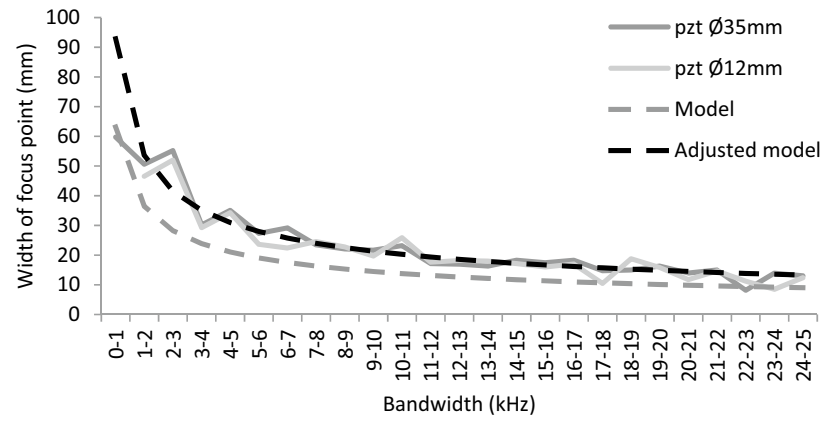

Fig. 5 Model and measurements of focus point's width on a $2 \mathrm{~mm}$ glass plate

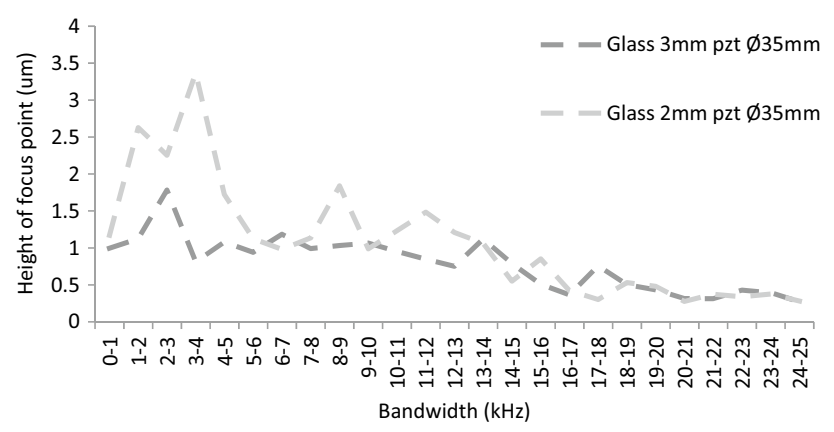

Fig. 6 Height of focus point $(\mu \mathrm{m})$ for the $35 \mathrm{~mm}$ transducers

model from Sect. 2 for the $3 \mathrm{~mm}$ thick plate. On Fig. 4, the focus point's width generated with PZT of both dimensions (12 and $35 \mathrm{~mm}$ ) on the $3 \mathrm{~mm}$ thick glass plate is plotted and compared to the computed analytical model of $R_{s}$. Both the model based on Eq. (6) and its adjusted counterpart, with the measured physical properties estimated with Eq. (10), are plotted. Overall the experimental data matches the adjusted analytical model. The different frequency intervals produced a spot varying from $60 \mathrm{~mm}(0-1 \mathrm{kHz})$ to $13 \mathrm{~mm}(24-25 \mathrm{kHz})$ corroborating the Kirchhoff based model calculations. Both experimental data curves exhibit similar variations.

On Fig. 5, the focus point's width generated with PZT of both dimensions ( 12 and $35 \mathrm{~mm}$ ) on the $2 \mathrm{~mm}$ thick glass plate is plotted. 12 and $35 \mathrm{~mm}$ piezoelectric diaphragms exhibit relatively close values to each other and to the adjusted model. The different frequency intervals produced a spot varying from $77 \mathrm{~mm}(0-1 \mathrm{kHz})$ to $15 \mathrm{~mm}(24-25 \mathrm{kHz})$, which is in good agreement to the Kirchhoff model. For both piezoelectric diaphragms' sizes the variation is similar.

In both cases the adjusted model with the measured physical properties shows a significant improvement in its prediction over the model without the adjustments.

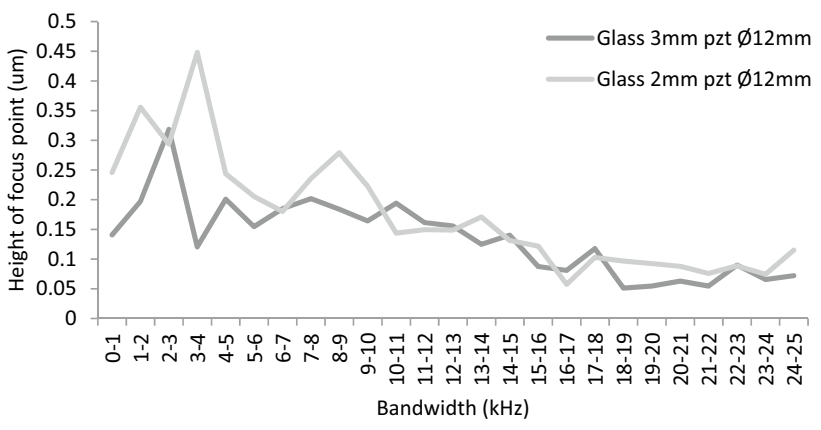

Fig. 7 Height of focus point $(\mu \mathrm{m})$ for the $12 \mathrm{~mm}$ transducers

\subsection{Focus point's height}

Figures 6 and 7 present the measurements for the focus point height, for each one of the four plates. The amplitude of the focalization varies strongly in the first half of the frequency domain for both plates with $35 \mathrm{~mm}$ wide PZT transducers. In the second half of the frequency domain a slow decrease is noted. For both plate thicknesses with $12 \mathrm{~mm}$ wide PZT transducers very similar variations were measured, albeit at a smaller scale.

Figure 8 presents scans of a selection of focus points from the $2 \mathrm{~mm}$ thick glass plate equipped with $35 \mathrm{~mm}$ PZT transducers. Depending on the bandwidth, the focusing does not always produce a perfect circular deformation. For example the focalization process in the $1-2 \mathrm{kHz}$ band produces a circular shape, whereas in the $3-4 \mathrm{kHz}$ it produces an ellipsoidal shape and in the $9-10 \mathrm{kHz}$ band it produces a triangular shape.

To better understand the behavior of the plate and the amplitude of the focus point, the frequency response at the focus point was measured for a $0-12.5 \mathrm{kHz}$ frequency sweep for the $2 \mathrm{~mm}$ thick plate equipped with $35 \mathrm{~mm}$ transducers and compared to the focus point height measurements in Fig. 9. A certain correlation between the density of high amplitude resonances and the focus point height can be observed, especially in the $3-4 \mathrm{kHz}$ and the $8-9 \mathrm{kHz}$ bandwidth.

Scans of the focus point region in the $3-4 \mathrm{kHz}$ were made to detect the resonant frequencies and the modal shape. Two major resonant frequencies (3.06 and $3.51 \mathrm{kHz})$ were observed, the corresponding modal shapes are shown in Fig. 10.

Scans of the focus point region in the $9-10 \mathrm{kHz}$ were also made. Two resonant frequencies $(9.28$ and $9.77 \mathrm{kHz})$ were observed, the corresponding modal shapes are shown in Fig. 11. 

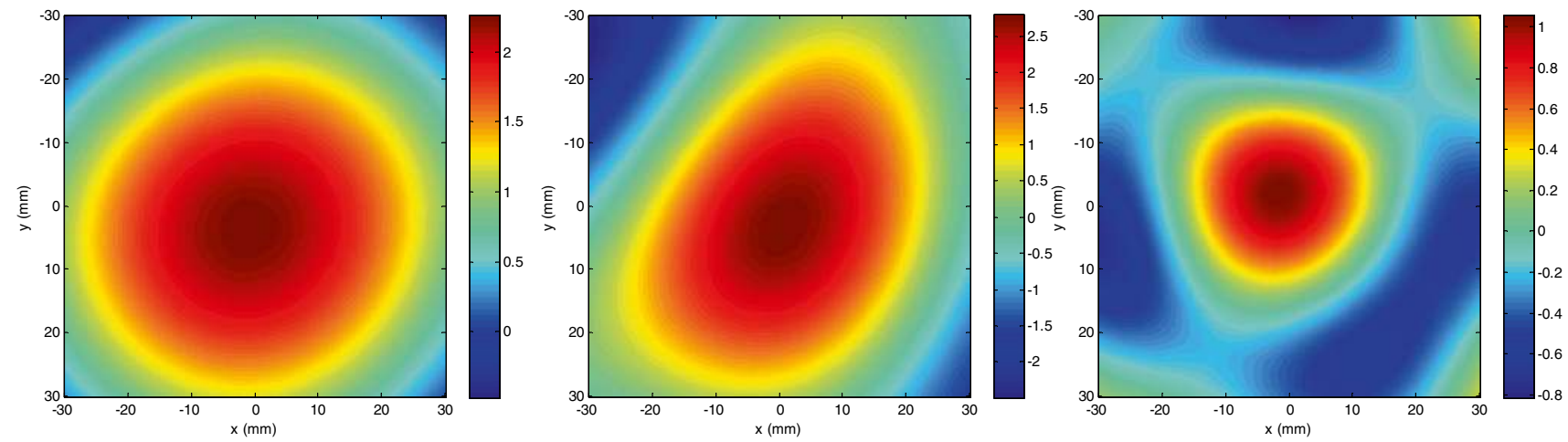

Fig. 8 1-2, 3-4 and 9-10 kHz bandwidth focus point's scan

Fig. 9 Focus spot height and frequency response comparison
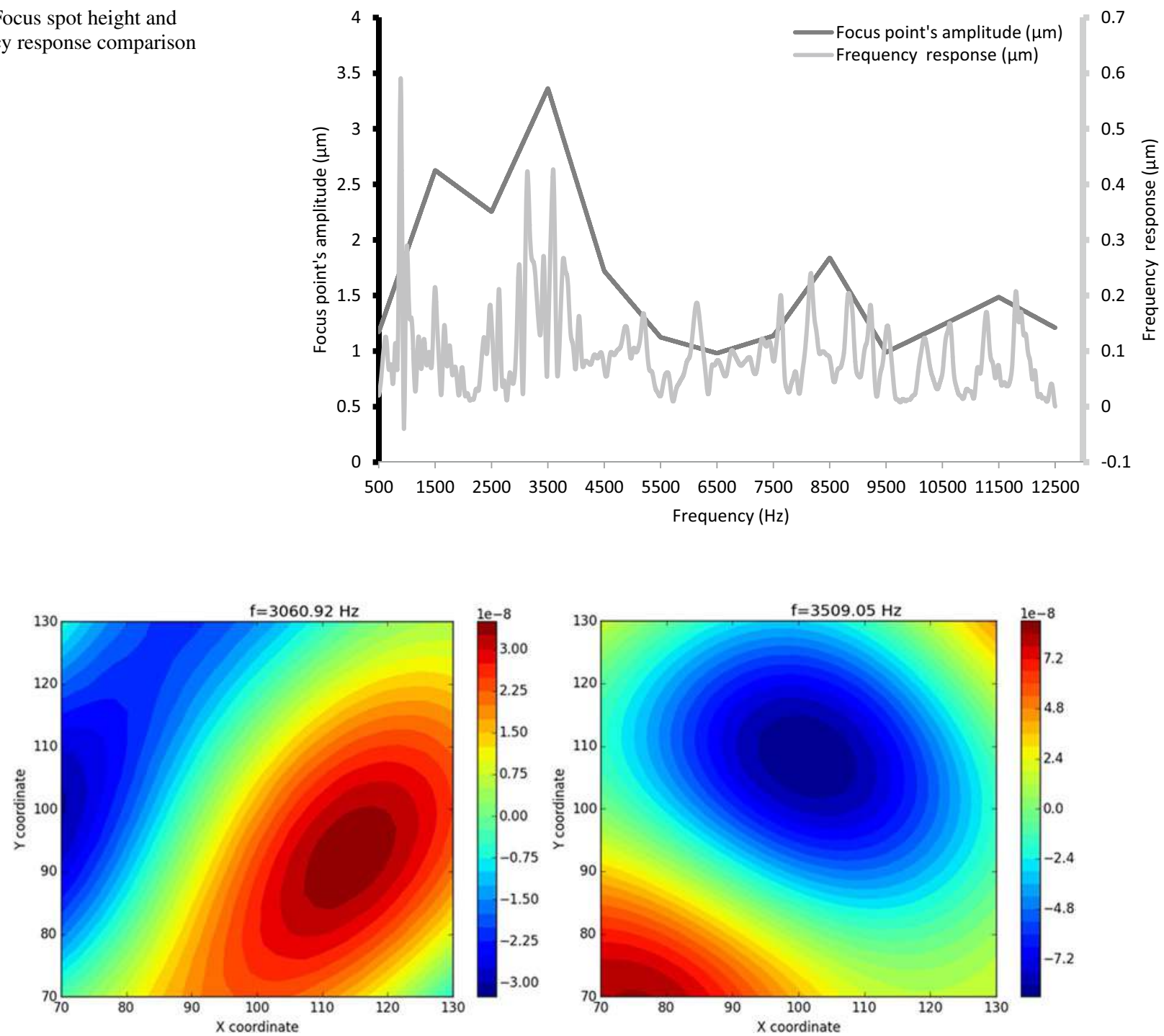

Fig. 10 Map of resonant frequencies in the $3-4 \mathrm{kHz}$ bandwidth for the $2 \mathrm{~mm}$ thick plate with $35 \mathrm{~mm}$ transducers 

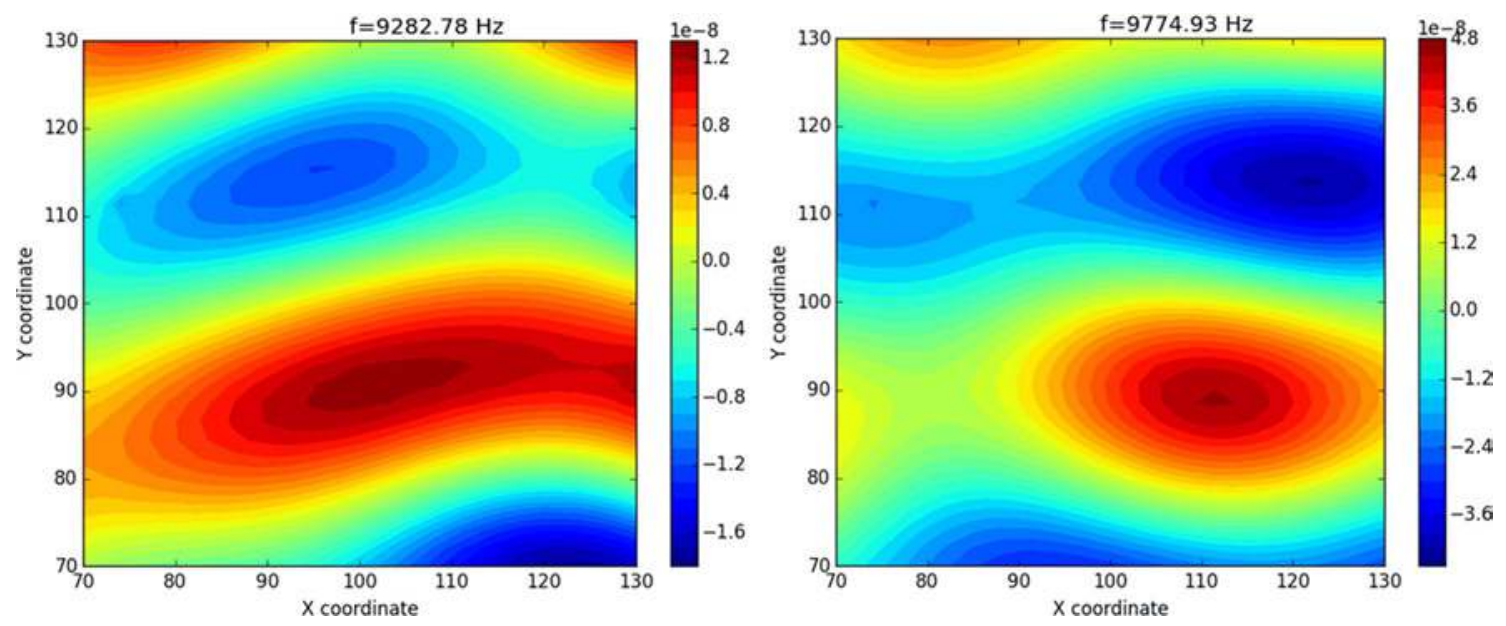

Fig. 11 Map of resonant frequencies in the $9-10 \mathrm{kHz}$ bandwidth for the $2 \mathrm{~mm}$ thick plate with $35 \mathrm{~mm}$ transducers

\section{Discussion}

Figures 4 and 5 show that for each glass thickness, the variations between 12 and $35 \mathrm{~mm}$ PZT transducers are small, meaning that the transducer's width has little influence on the achievable spatial resolution. The main parameters to determine the focus point's width are the mean frequency of the excitation bandwidth and the plate's geometry and material according to the model based on Kirchhoff theory. This was verified on $1 \mathrm{kHz}$ frequency bands.

The amplitude of the focus point was measured in each configuration. Strong variations were observed in the lower frequency domain $(0-1 \mathrm{kHz}$ until 9-10 kHz). Measurements of the system's frequency response at the exact location of the focus spot show a correlation between the density of high amplitude resonances and the focus point height, especially in the $3-4 \mathrm{kHz}$ and the $8-9 \mathrm{kHz}$ bandwidth.

It was noted that for certain frequency bands, the focus point was not perfectly circular, unlike in the case of a wide bandwidth excitation signal. The time reversal process adds every contribution from every frequency across the whole frequency bandwidth. However the modal shapes inside narrow bandwidth excitation signals could dominate in the time reversal process and explain the distortion observed in some cases.

According to the model, and confirmed by the measurements, spatial resolution increases the same way as the mean frequency of the excitation signal band is increased for given plate characteristics (material and geometry). Targeting a spatial resolution of $10 \mathrm{~mm}$ requires a mean frequency of the excitation signal around $50 \mathrm{kHz}$ on the $2 \mathrm{~mm}$ thick plate. But the higher the frequency, the more difficult it is to drive the transducers in order to produce such high frequency waves in the plate as the energy consumption increases.
As seen with measurements, amplitude decreases when excitation frequencies increase. In order to get sufficient amplitude, lower frequencies should be used. For the $2 \mathrm{~mm}$ plate, the contribution of the $0-10 \mathrm{kHz}$ is predominant. Nevertheless, the hearing range of humans is from 20 to $20 \mathrm{kHz}$. When generating sufficiently big deformations of the plate in this frequency range being able to recognize while exploring the surface with the finger, noise is emitted that alters user acceptance.

Another important matter is the contrast ratio between the peak amplitude of the focus point and the mean amplitude of the waves in the plates. The higher the ratio, the easier it is to distinguish the stimulus. Two parameters are fundamental for the contrast ratio: the signal's bandwidth and the number of transducers (Hudin et al. 2012). However, the wider the bandwidth, the harder it is to build an electronic with a flat response across the whole band. The number of transducers is easier to increase within a certain limit.

For a given application, the only geometrical parameter which can be modified is the plate's thickness. The plate's surface matches of course the surface of the screen. The model shows that with the decrease in thickness, the spatial resolution gets higher. But more importantly, the amplitude of the focus point is improved by a thinner plate.

\section{Conclusion}

This paper investigated the factors influencing the dimensions of a focus point obtained through time reversal of acoustic waves. The mean frequency of the bandwidth influences the width of the focus point and the plate's thickness influences primarily the displacement amplitude of the spot. Different sizes of piezoelectric diaphragms (12 and 
$35 \mathrm{~mm}$ ) did not yield significant differences on the focus point dimensions ( $8 \%$ in average). The resonant behavior of the plate and its implication on focus point dimension, in particular its shape and amplitude are investigated. On reduced bandwidths ( $1 \mathrm{kHz}$ wide) the predominant effect of the resonant frequencies on the amplitude and shape of the focus spot is shown.

\section{References}

Aki K (1957) Space and time spectra of stationary stochastic waves, with special reference to microtremors

Boschi L et al (2013) On measuring surface wave phase velocity from station-station cross-correlation of ambient signal. Geophys J Int 192:346-358

Cassereau D, Fink M (1992) Time-reversal of ultrasonic fields. III. Theory of the closed time-reversal cavity. IEEE Trans Ultrason Ferroelectr Freq Control 579-592

Chen H-Y, Park J, Dai S, Tan H (2011) Design and evaluation of identifiable key-click signals for mobile devices. IEEE Trans Haptics 229-241

Fink M (1992) Time reversal of ultrasonic fields. I. Basic principles. IEEE Trans Ultrason Ferroelectr Freq Control 39:555-566
Fink M, Prada C (2001) Acoustic time-reversal mirrors, Inverse Prob $17(1)$

Hoggan E, Brewster SA, Johnston J (2008) Investigating the effectiveness of tactile feedback for mobile touchscreens. ACM, New York, pp 1573-1582

Hudin C (2014) Focalisation par retournement temporel dans les plaques minces: application à la stimulation tactile, Université Pierre et Marie Curie-Paris VI

Hudin C, Lozada J, Wiertlewski M, Hayward V (2012) Tradeoffs in the application of time-reversed acoustics to tactile stimulation. Haptics: perception, devices, mobility, and communication. Springer, Berlin, pp 218-226

Hudin C, Lozada J, Hayward V (2013) Localized tactile stimulation by time-reversal of flexural waves: case study with a thin sheet of glass. 67-72

Kildal J (2012) Kooboh: variable tangible properties in a handheld haptic-illusion box, Haptics: perception, devices, mobility, and communication. Springer, Berlin, pp 191-194

Mortimer BJP, Zets GA, Cholewiak RW (2007) Vibrotactile transduction and transducers. Acoust Soc Am J 121:2970

Poupyrev I, Maruyama S, Rekimoto J (2002) Ambient touch: designing tactile interfaces for handheld devices. ACM, New York, pp $51-60$

Wu F, Thomas J-L, Fink M (1992) Time reversal of ultrasonic fields. II. Experimental results. IEEE Trans Ultrason Ferroelectr Freq Control 39:567-578 